\title{
3. INTRODUCTION
}

The health status of children is influenced by family, social, and economic factors. Information about these influences on child health has not been routinely collected. ${ }^{1,2,3}$ Since most children are healthy, hospital admissions and other administrative data do not provide a good overall picture of child health and wellbeing.,4 Nor do routine data collections provide information on childhood risk factors for diseases and ill health presenting in adulthood. Identification of risk factors is important for formulation of programs for health promotion and protection. The New South Wales Child Health Survey took into account these information deficits and incorporated state and national child health information priorities. It provides the first broad overview of the health and wellbeing of children and families in NSW.
This supplementary issue of the NSW Public Health Bulletin provides information on the development of the New South Wales Child Health Survey including the consultation process, the development of the survey instrument, and the development of survey methods. It is important to note that the process of developing the Child Health Survey was iterative in nature rather than being divided into distinctive steps. More information on results and final methods used in the Child Health Survey can be accessed by contacting the NSW Health Survey Program at the NSW Department of Health or by visiting the following website: www.health.nsw.gov.au/public-health/ phb/phb.html. 